\title{
Experimental Study on Poultices Applying to Remove Fixative (Paraloid B72) on Earthen Mural Painting
}

\author{
Kyeong Min Lee | Hye Young Moon | Yeong Gyeong Yu* | Soon Kwan Kim ${ }^{1}$ \\ Cultural Heritage Conservation Science Center, National Research Institute of Cultural Heritage, \\ Daejeon, 34122, Korea \\ *Department of Cultural Heritage Science, Chungbuk National University, Cheongju, 28116, Korea \\ ${ }^{1}$ Corresponding Author: 1km614@korea.kr, +82-42-860-9377
}

\begin{abstract}
The possibility of applying poulticing was studied for removing Paraloid B72, a resin used for fixing an earthen mural painting. Five types of poultices were selected from clay and gel types, and acetone, ethanol, and methyl ethyl ketone(MEK) were used as mixed solvents. The possibility of mixing between the poultice and solvent was investigated, and then the spreadability, fluidity, acidity, drying properties, and solubility of the poultices were examined to confirm the characteristics. A poultice agent, which is suitable for applying to a mural painting, was selected and applied to a painting layer sample coated with Paraloid B72. As a result, all painting layers were good condition at under $50 \%$ of the solvent. The removal efficiency of Paraloid B 72 was more effective when most of the solvents were used at 50\%, rather than at $25 \%$. However, it is difficult to mix $50 \%$ of MEK and ethanol with the gel-type poultices. When used at $25 \%$, the removal performance was poorer than that at $50 \%$, but the mixing with all the poultices was successful. In addition, the adsorption and removal power of the gel-type poultice were better than those of the clay-type, but the latter was expected to be more suitable in short-time cases due to its high drying speed. The results of this study show that the dissolution performance varies depending on the poultices and solvents. Hence, the poultice and solvent should be selectively applied, considering the fixative of the mural to be removed.
\end{abstract}

Key Words: Earthen mural painting, Paraloid B72, Poultice, Gelling, Solvent

\section{INTRODUCTION}

The conservation of Buddhist mural paintings in Korea has commenced in full gear since the early 1980s when synthetic resins were used back then, followed by acrylic resins during the period between the 1980s and the 1990s. Between the 1990s and the early 2000s vinyl acetate resins used during the period before adhesives used to natural materials in the mid-2000s. In particular, with the introduction of the characteristics of fixatives announced by the Istituto Superiore per la Conservazione ed il Restauro(ISCR), Italy in the early 1990s, Paraloid B72 was widely used for the conservation purpose of properties made of inorganic materials and was also used as a surface consolidating agent for Buddhist mural paintings in temples as well(Han, 2002).
In recent years, validation studies have been conducted to apply a fixing agent suitable for the coloring layer fixing treatment of mural paintings made of clay in the Joseon Dynasty(Lee et al., 2013).

The investigation of the conditions of the Buddhist mural paintings in temple along with studies on the deterioration of color layers following the 2000s has found accelerating damage on the earthen mural paintings with the application of Paraloid B72 for the fixation purpose due to the exposure to the external environment characterized by ultraviolet rays and humidity for an extended period of time (Lee et al., 2008). The Buddhist Mural painting of Buseoksa temple and Buddhist mural painting of Shinheongsa temple among earthen mural paintings that adopted Paraloid B72 as a conservative agent, for example, are also 
reported to show a variety of physical damages, including layer-exfoliation(National Research Institute of Cultural Heritage, 2015). Acrylic resins feature extremely low reversibility after being hardened, and it is nearly impossible to remove the resin applied to the mural paintings while fully ensuring the stability of the color layers. However, the investigation so far indicates that the synthetic adhesive fixative causes the increasing deterioration of the color layers over time due to the properties of materials used in earthen mural paintings and the effects of the surrounding environment, necessitating further studies on how to remove it and to stabilize the condition of the paintings.

Several studies in Korea have found that the poulticing treatment is effective in removing contaminants from various cultural properties, including ceramics and stone cultural artifacts and that it has actually been used in cultural properties at home and abroad made of a variety of materials, including wood, paper, and leather. However, the applicability of the poultice to earthen mural paintings has yet to be subject to in-depth studies in Korea. Earthen mural paintings feature softness compared to the properties of other inorganic materials with many things to take into consideration in applying the poulticing treatment method, including the vertical erection on the wall of a building. In addition, to properly remove the synthetic resins adhered to the surface of earthen mural paintings that may cause the damage on the cultural properties of solvent and poultice, a solvent must be used, which require a prior investigation of various properties, including the mixing performance and solvency depending on their types. In this study, we evaluate the applicability of poulticing for the removal of Paraloid B72 applied to the mural painting by examining the suitable manufacturing conditions of the poultice, the effect and stability as a remover.

\section{RESEARCH BACKGROUND AND TRENDS}

The poulticing treatment is a method of removing contaminants such as stains or undesired materials from the surface of valuable cultural properties in a controlled manner using soft, moist, hygroscopic and viscous mass on the surface. The poultice material can be made of various substances, including soft fiber, gel, and clay, various combinations of poultices and solvents depending on the nature of cultural properties subject to conservation and the type of contaminants to be removed(American Institute for Conservation of Art and Historic Works, 2018). The principle of poulticing is that contaminants dissolved by a solvent are removed as they are dried and moved to the poultice.

One of the advantages of poulticing is that it can be used in an intended local area only with easy workability. In addition, the organic solvent used mainly to removing contaminants is highly volatile and its drying speed can be controlled if used together with the poultice if surface treatment on the contaminated area is difficult.

\subsection{Domestic trends}

In Korea, poulticing has been adopted for the purpose of removing surface contaminants on stone and ceramic cultural properties. Outdoor stone properties are often damaged by graffiti using paints and lacquers, and the graffiti on the 'Samjeondobi' in Seoul that occurred in 2007 was created using a commercial spray-type red lacquer. An organic solvent effective in removing target surface contaminants was carefully selected and was mixed with methyl cellulose to remove contaminants repeatedly using the adhesive poulticing method. The remaining fine residues were removed by repeatedly applying laponite, a water-soluble gel-type agent(Lee et al., 2009). The characteristics of the poulticing treatment applied to stone cultural properties is that it has the surface of the rock soaked in distilled water to prevent the contaminants dissolved in organic solvent from spreading around the rock, and the poultice is sealed with the polyethylene film to treat the surface with a sufficient drying time of some 24 to 48 hours, in general.

The poulticing treatment using a mixture of solvent and poultice has turned out to be effective in removing metal corrosion existing in the form of stain on the surface of the ceramics excavated underground. When it comes to using the poulticing treatment, however, careful attention is required in determining the number of applications, 
duration of application, and the concentration depending on the conditions of cultural properties and/or contaminants (Park et al., 2011), on which there are few studies in Korea. In 2017, a study was announced as to the poulticing treatment using a poultice material mixed with an organic solvent as a surface conservation agent to properly remove Paraloid B72 from the surface of soil mural paintings. It has been confirmed that the organic solvents whose solubility for Paraloid B72 was confirmed selected and mixed with activated carbon fibers and methyl cellulose, which are poultice materials, is effective in removing contaminants from the color layers and stabilizing the surface(Jin et al., 2017). The materials used as adsorbents served as media for mixing with an organic solvent, which is not entirely considered the poulticing treatment.

\subsection{Overseas trends}

In Korea, studies have been conducted on materials suitable for the poulticing treatment on ceramics and stones featuring solid, hard surface. A number of overseas studies have been carried out as to the application of the poulticing treatment to a more variety of materials, including limestone carvings, lime wall paintings, leathers, papers and, wooden furniture. The studies have been extended to include different types of contaminants to be removed, including deteriorated varnish layers, label adhesives on leather, masking tapes, glues, and Paraloid B72. The most commonly used poultices are found to be laponite, methyl cellulose, and carbopol. When it comes to organic solvents featuring solubility of contaminants to be removed, methyl ethyl ketone(MEK) and ethyl acetate are most commonly used for removing Paraloid B72, acetone for masking tapes and acetone, ethanol and ethyl acetate for label adhesives on leather, and acetone, ethyl acetate and xylene for the varnish layer with the concentration of mostly 20 to $50 \%$. Laponite is successfully gelled using deionized water and is mixed with solvent, followed by the facing treatment to remove residuals from the surface of cultural properties with Japanese paper, rayon paper, and cotton used as facing materials. In addition, the processing time of application to organic materials such as leather or paper is found to be several minutes to several tens of minutes as opposed to the application to ceramics, lime walls, etc., which usually takes as long as several hours(Warda et al., 2007; Garland and Twilley, 2010; Deurenberg, 2013; O'Hern and Pearlstein, 2013; Tong, 2013; Brajer et al., 2014).

\section{MATERIALS AND METHOD}

\subsection{Characteristics of poultices}

\subsubsection{Materials for experiments}

The poultice comes in different types: gel, clay and fiber, among which clay and gel types have been selected considering that it has to be fixate for a certain period of time when attached to the surface of mural paintings. A total of five poultices have been selected as experimental materials: Bentonite and sepiolite in clay type, which have been used for removing contaminants from ceramics, laponite, which is the most commonly used gel-type poultice, and carbopol and methyl cellulose in gel-type, which are known to be mixable with organic solvent. Paraloid B72 is an acrylic copolymer resin with ethyl methacrylate and is soluble in acetone, ethanol, xylene, toluene, and methyl ethyl ketone(hereinafter referred to as 'MEK'). Preliminary experiments have found that toluene and xylene, which are non-polar solvents won't be mixed with bentonite, carbopol, laponite, and methyl cellulose, or that the poultice won't be gelled. Therefore, the study has selected three agents of acetone, ethanol and MEK and examined their characteristics after mixing with the poultice.

\subsubsection{Experiment methods}

\section{Workability test}

This study has taken the suggestions of previous studies as to how to mix a poultice with an organic solvent with the latter's concentration set at two versions: 50\% (high concentration) and $25 \%$ (low concentration) in consideration of worker's safety and to be able to compare contaminant removal performance. The test has been conducted to identify if the completed poultice feature easy conservation and the hanging test has been carried out to confirm the fluidity when applied to the vertical mural painting surface. 


\section{$\operatorname{Acidity}(\mathrm{pH})$ measurements}

The study has investigated the physical properties of those in experiment groups except those that failed to be gelled in mixture with organic solvents. The acidity of the poultice has been measured to ensure the stability of the color layers when applying the poulticing treatment to the surface of cultural properties. the poulticing materials were mixed with deionized water to measure its own acidity.

\section{Drying of organic solvents}

The weight measurements have been made in $3,6,12$ and 24 hours after the application of the poultice to the sample to identify any different in drying aspects.

\section{Solubility}

Experiments have been conducted to determine the solubility of Paraloid B72 after organic solvent is mixed with poultice. Paraloid B72 at a concentration $10 \%$ has been applied to the aluminum sample twice with xylene used as a solvent. Since the coating layer of Paraloid B72 is colorless and transparent, a small amount of Seokganju has been added to facilitate the observation before and after removal. To identify and compare the degree of changes upon removal, a chromaticity measurement, microscopic examination, and FT-IR/IR have been conducted on a single area of the Paraloid B72 coating layers.

\subsection{Applying removal fixative}

\subsubsection{Materials}

Upon identification of poultices suitable for the poulticing treatment and the organic solvents that can be mixed, the pseudo-sample have been prepared for the application test to confirm the effect of removing contaminants and the stability of color layers when applying them to the color layers of mural paintings. The pseudo-sample have been prepared by mixing clay and sand at a ratio of $1: 2$ in reference to the soil components and ratios of the finishing layer of soil mural painting and have been thoroughly dried at room temperature before preparing the color layer using mineral pigment glue and animal glue. The pigment has been prepared using two different pigments: mineral pigment of Seokrok(malachite) and soil pigment of Seokganju(red ocher) with an animal glue used as a base and has been used for coloring twice. A $10 \%$ solution of Paraloid B72(in xylene) has been applied three times to the top of the dried color layer. A total of six test samples found to be suitable for the poulticing treatment based on the poultice characteristics test with an appropriate level of removal effect and low residual color layer have been selected and applied to the surface of Paraloid B72. They include LP_AH and LP_AL for laponite, LP_ML for laponite, $\mathrm{MC} \_\mathrm{EH}$ and $\mathrm{MC} \_\mathrm{AL}$ for methyl cellulose and SE EH for sepiolite.

When applying the poulticing treatment, the rayon faced in two layers has been applied to protect the color layers. Different application times have been set according to the type of poultice: 3 hours for clay type and 6 hours for gel type. The surface of poultice has been covered with polyethylene vinyl for the duration of application.

\subsubsection{Method}

In a bid to compare the color layers before and after removal of Paraloid B72, the poultice has been applied to the surface of original color layer and the color layer applied with Paraloid B72, followed by a series of inspections and measurements, including visual inspection, microscopic inspection, chromaticity measurement, and contact angle measurement on the surface of the color layers after the removal of the synthetic resin.

The condition of color layers by specimen has been visually observed and has been recorded through digital photographing. The microscopic inspection with 200 magnifications has been conducted on the same spot of each specimen to examine the presence of the pigment particles and the surface condition in minute detail. The chromaticity of three randomly selected spots of a specimen (left, right and center of each specimen) has been measured, followed by the comparison of color differences before and after the application. Another comparison of contact angles has been made for the random selected spots of each sample before and after the application of each sample. 


\section{RESULTS}

\subsection{Evaluation of characteristics of properties}

\subsubsection{Workability test}

Both tests have found that bentonite and sepiolite are well mixed with all solvents and feature excellent workability in terms of fluidity in poultice method. The successfully gelled acetone and ethanol, MEK in low concentration and gel-type poultice mixtures have also shown to feature excellent workability due to their superior adhesion and very low fluidity found in the vertical surface treatment test. It is failed to be gelled that laponite, carbopol, methyl cellulose were made of MEK in high concentration. methyl cellulose with MEK in low concentration is also failed. The tests have also found that laponite is gelled at low concentrations only but not at high concentrations(Table 1,2).

\subsubsection{Acidity $(\mathrm{pH})$ measurements}

Bentonite and sepiolite have shown to be weakly alkaline with 9.8 and 8.32 , respectively and laponite also to be weakly alkaline with 10.02 as well. Carbopol and methyl

Table 1. Results of applying test

\begin{tabular}{|c|c|c|c|c|c|c|c|c|c|c|}
\hline Poultice & \multicolumn{2}{|c|}{ Bentonite } & \multicolumn{2}{|c|}{ Sepiolite } & \multicolumn{2}{|c|}{ Laponite } & \multicolumn{2}{|c|}{ Carbopol } & \multicolumn{2}{|c|}{ Methyl cellulose } \\
\hline Ratio & $1: 1$ & $1: 3$ & $1: 1$ & $1: 3$ & $1: 1$ & $1: 3$ & $1: 1$ & $1: 3$ & $1: 1$ & $1: 3$ \\
\hline Acetone & & & & & & & & & & 53 \\
\hline Ethanol & & & & & & & & & & \\
\hline $\begin{array}{l}\text { Methyl ethyl ke } \\
\text { (MEK) }\end{array}$ & & & & & & & & & & \\
\hline
\end{tabular}

Table 2. Results of hanging test

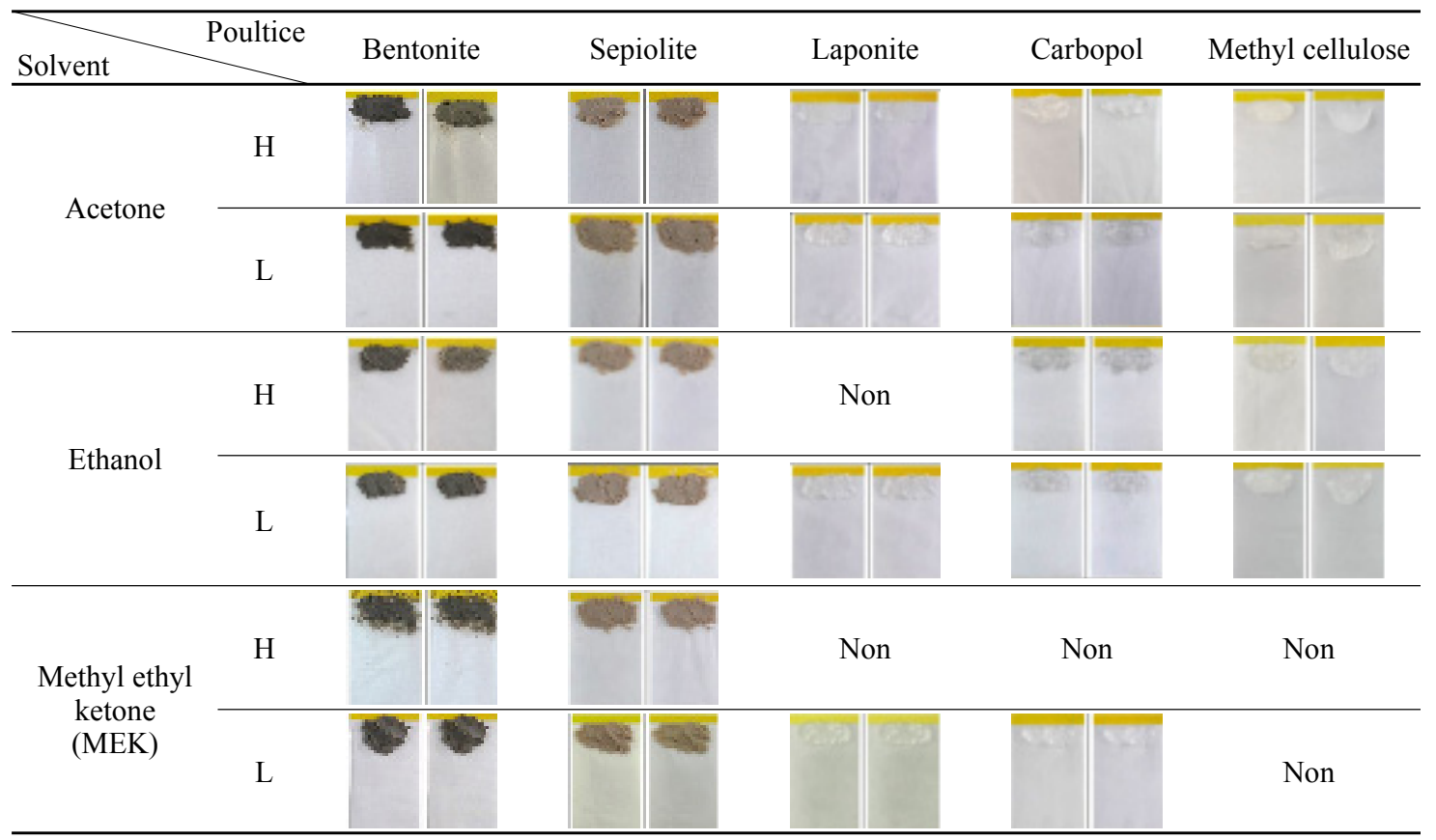


cellulose were found to be close to neutrality with 6.87 and 6.57 , respectively. When it comes to the acidity of poultices mixed with organic solvents, both bentonite and sepiolite were found to be alkaline with its acidity ranging from 9.75 to 10.72 and 7.75 to 8.4 , respectively similar to the acidity of the raw materials. Laponite was also found to be weakly alkaline with its acidity ranging from 10.39 to 10.6 , similar to that of the raw materials. Methyl cellulose was 6.61 7.32, which was neutral and similar to the raw material. Carbopol have shown different acidities depending on the solvent's concentration with acetone and ethanol mixed at high concentrations shown to be acidic with the acidity of 4.7 and 4.98, respectively. Those in low concentration group were close to neutrality with their acidities ranging from 6.27 to 6.95 . Carbopol mixed with acetone and ethanol in high concentrations was shown to be acidic, resulting in exclusion from further experiments as it is not suitable for surface treatment for conservation
purposes(Figure 1).

\subsubsection{Drying of organic solvents}

There have been more significant changes in weight among samples in high concentrations than those in low concentrations with the largest weight change in MEK, followed by acetone and ethanol. Acetone and MEK saw the highest weight changes among those in clay type for less than six hours while acetone had the highest among those in gel type. Ethanol saw a constant weight change over time in both clay and gel types. In addition, all samples saw a higher amount of solvent evaporation among those in clay type than those in gel type during the same period of time(Figure 2, 3).

\subsubsection{Solubility}

MEK has shown to have the highest removal efficiency for Paraloid B72, followed by ethanol and acetone. When it

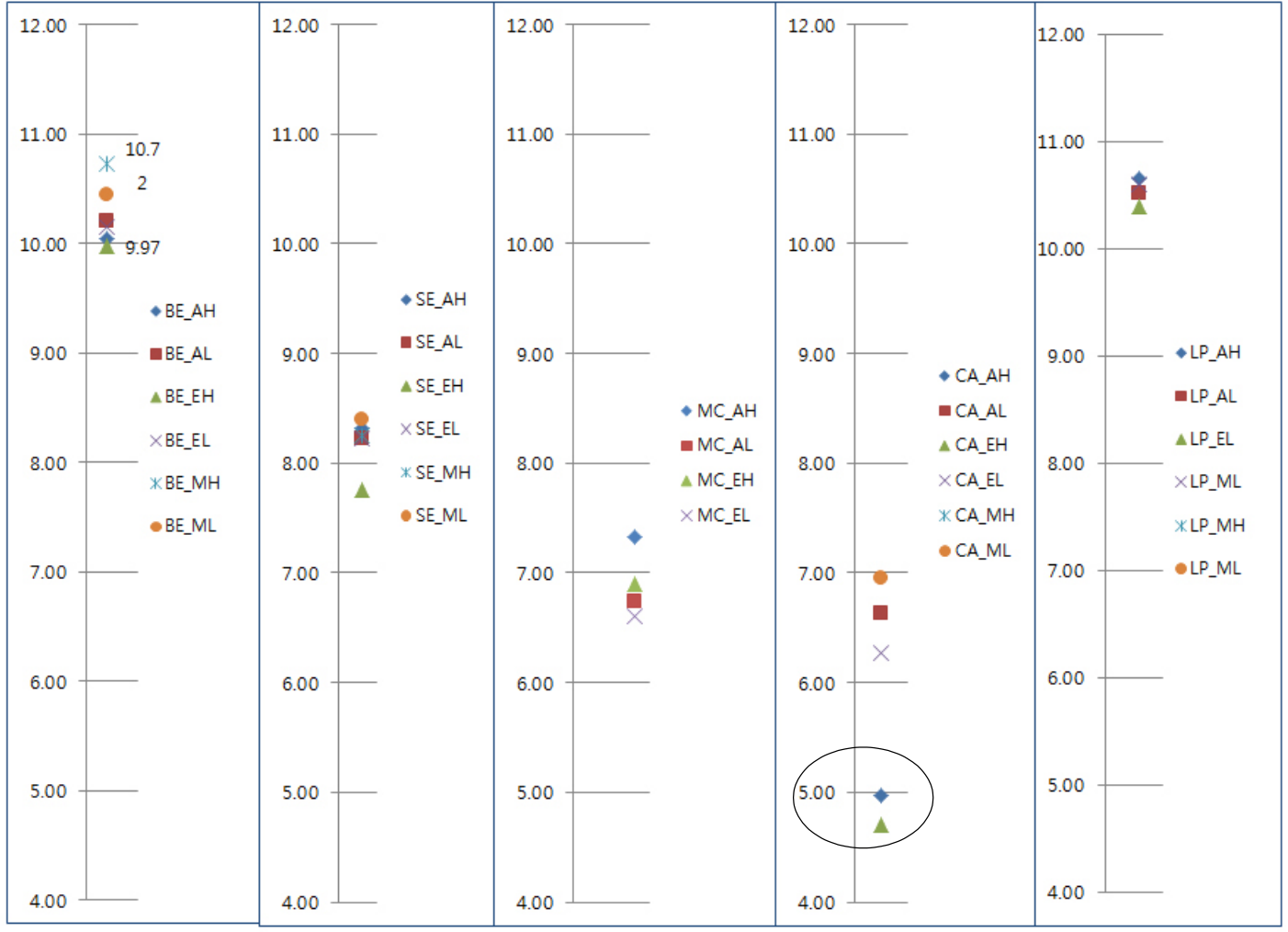

Figure 1. Results of acid degree for poultices. 
comes to the difference of removal efficiency between high concentration and low concentration, MEK and ethanol have shown to have a certain degree of difference but acetone has shown little difference. The removal efficiencies of some solvents have been higher in methyl cellulose and laponite, which come in gel type than those of bentonite and sepiolite. Acetone saw a negligible degree of change among those in clay type but a clear sign of removal in those in gel type. However, those in gel type saw a spilling of solvent around the application area and carbopol, in
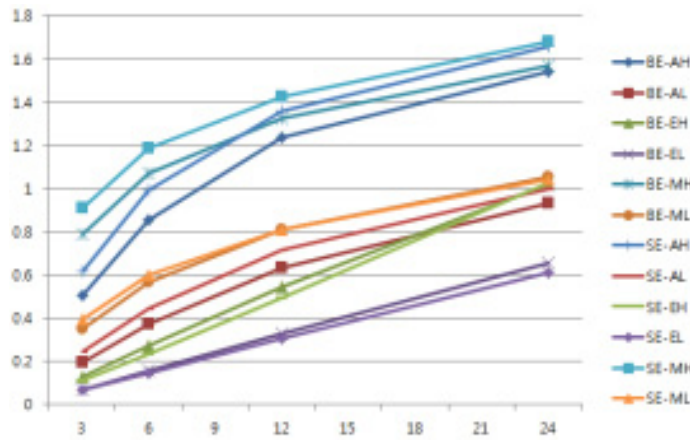

Figure 2. Weight reduction of clay type poultices. particular, saw the leftover of poultice material(Table 3).

The analysis has been conducted except completely removed sample such as FT-IR MEK. When comparing the spectra, the characteristic peak $\left(1700 \mathrm{~cm}^{-1}, 2300 \mathrm{~cm}^{-1}\right)$ of Paraloid B72 and the trabecular characteristic peak $\left(1164 \mathrm{~cm}^{-1}\right.$, $1029 \mathrm{~cm}^{-1}$ ) of Paraloid B72 have been found to decrease to some extent upon removal(Figure 4, 5), and carbopolacetone and methyl cellulose-ethanol specimen have shown to have peaks similar to those of carbopol and methyl cellulose, which indicates the presence of residuals (Figure 6, 7).

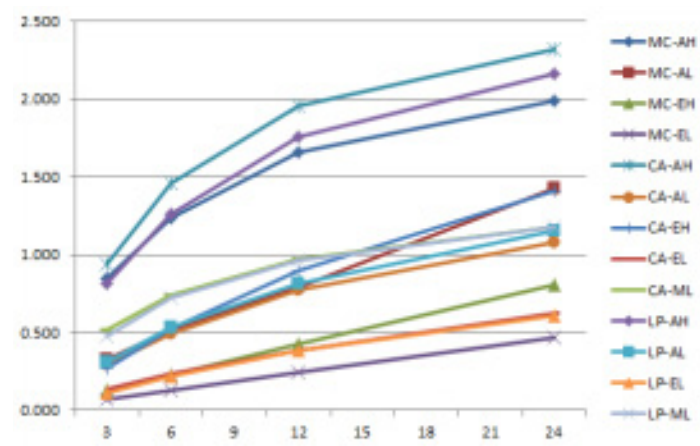

Figure 3. Weight reduction of gel type poultices.

Table 3. Results of removing test

Solvent Poultice
Acetone
(Left: H,
Right: L)

$(* \mathrm{H}$ : High concentration, L: Low concentration) 

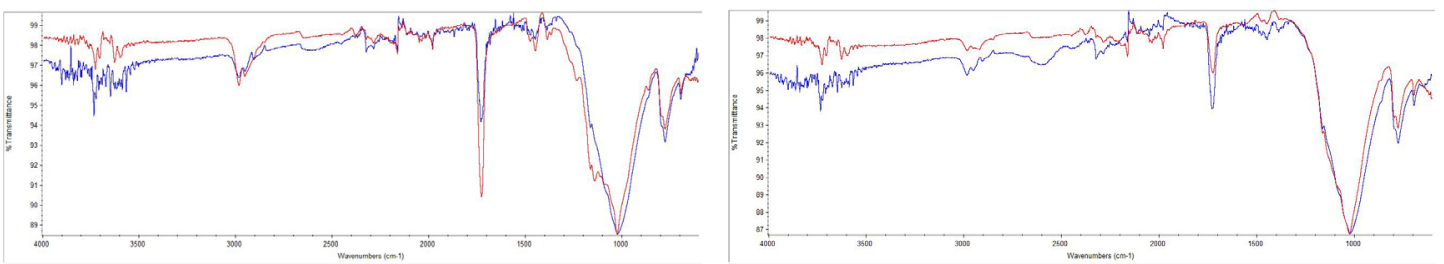

Figure 4. FT-IR spectrum of LP_AL(Before: blue, After: red). Figure 5. FT-IR spectrum of CA_ML(Before: blue, After: red).

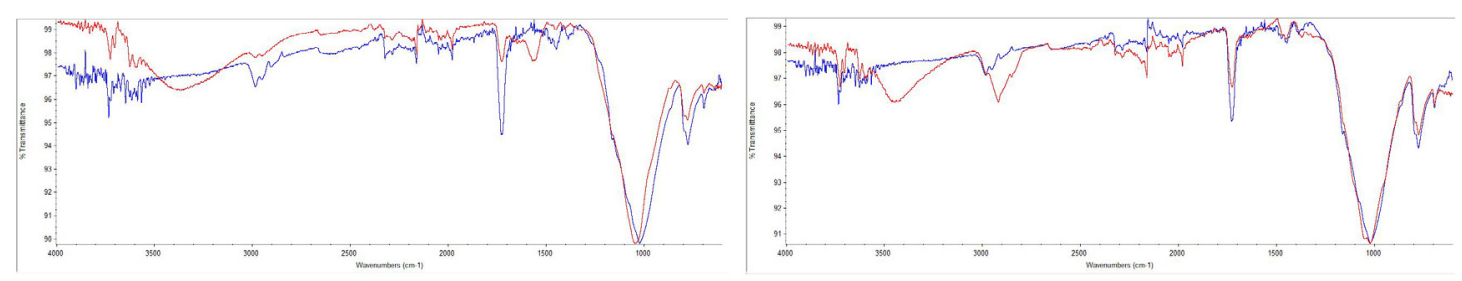

Figure 6. FT-IR spectrum of CA_AL(Before: blue, After: red). Figure 7. FT-IR spectrum of MC_EL(Before: blue, After: red).

Table 4. Microscope observation of Paraloid B72 removed sample

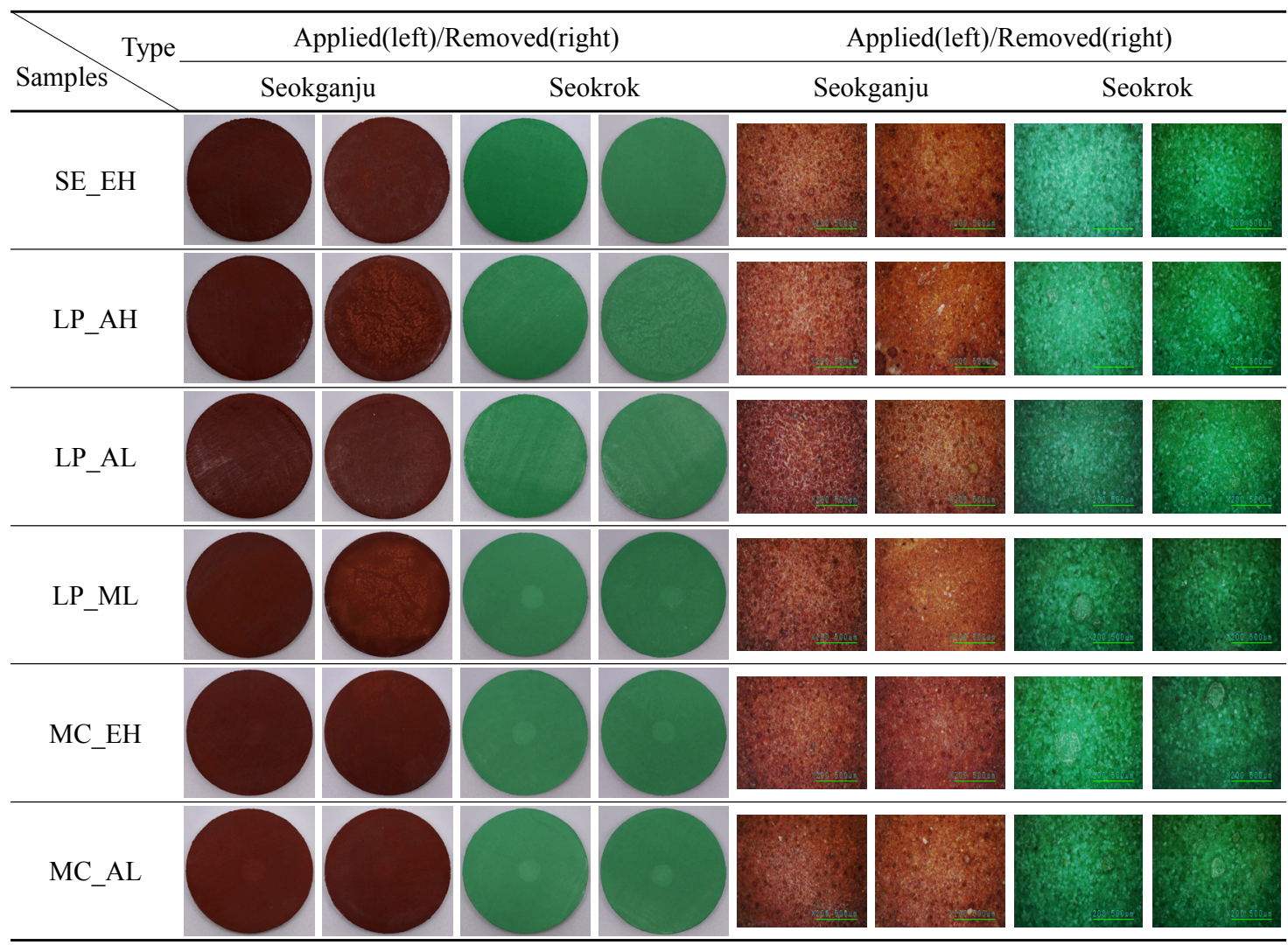

(*SE: Sepiolite, EH: Ethanol high concentration, LP: Laponite, AH: Acetone high concentration, AL: Acetone low concentration, ML: MEK low concentration, MC: Methyl cellulose) 


\subsection{Applying removal fixative}

\subsubsection{Results of visual and microscopic inspections}

The LP_AH, LP_AL, MC_AL, MC_EH, and SE_EH in experiment group have shown no traces of spilling or smearing of pigment over the facing surface and the poultice. However, the LP_ML have shown some physical damage on the color layer due to the reattachment between the fixative and the facing material caused by the dissolution of Paraloid B72 although it didn't have any damage caused by the application of solvent such as spilling of pigment. LP_AH and LP_ML have showed the most significant removal effect. LP_AL and SE_EH have also shown some removal effect but with no clear signs except the trace of dissolution only. The visual inspection of $\mathrm{MC} \mathrm{AL}$ and $\mathrm{MC} \mathrm{EH}$ has found no significant changes. The microscopic inspection has clearly confirmed the degree of dissolution of Paraloid B72(DG-3, SCALAR, Japan $)(\times 200)$ LP_AH has shown to have the transparent coating layer created by the dissolution of Paraloid B72 completely gone, except the dissolved resins observed as remnants in the form of thread in some sample. The
LP_ML have seen Paraloid B72 dissolved but the color layers of Seokganju were uneven, resulting in some areas observed to be brighter like stains.

LP_AL and SE_EH have not seen Paraloid B72 completely removed except that its coating layer has been observed to be more dissolved after the application. No thread-from remnants have been observed in LP AH. The MC AL have also shown to have the Paraloid B72 coating layer slightly degraded according to the microscopic inspection(Table 4).

\subsubsection{Results of color change}

The Seokganju sample and seokrok sample have seen a change of color with the average $\Delta \mathrm{E}$ of approximately 10 to 12 and 3 to 4, respectively upon the application of Paraloid B72. The LP_AH sample, which have shown to have the highest removal effect based on the visual inspection have seen a change of color with the average $\Delta \mathrm{E}$ of Seokganju approximately 3.6 to 3.9 after the poulticing treatment with just as much color change from the original sample as well. The LP_AL and SE_EH have seen a change of color for Seokganju with the average $\Delta \mathrm{E}$ of approximately 1.5 and 1 to 2 , respectively upon application of the poulticing

Table 5. Colour difference of samples

\begin{tabular}{ccccccc}
\hline \multirow{2}{*}{ Samples } & \multicolumn{3}{c}{ Seokganju } & \multicolumn{3}{c}{ Seokrok } \\
\cline { 2 - 7 } & $\begin{array}{c}\text { Original } \sim \\
\text { applied }\end{array}$ & $\begin{array}{c}\text { Original } \sim \\
\text { removed }\end{array}$ & $\begin{array}{c}\text { Applied } ~ \\
\text { removed }\end{array}$ & $\begin{array}{c}\text { Original } \sim \\
\text { applied }\end{array}$ & $\begin{array}{c}\text { Original } \sim \\
\text { removed }\end{array}$ & $\begin{array}{c}\text { Applied } ~ \\
\text { removed }\end{array}$ \\
\hline SE_EH & 12.55 & 11.60 & 1.40 & 3.90 & 3.99 & 0.45 \\
\hline LP_AH & 12.67 & 9.46 & 3.73 & 3.22 & 3.76 & 0.71 \\
\hline LP_AL & 12.87 & 12.54 & 1.54 & 3.56 & 4.10 & 0.66 \\
\hline LP_ML & 12.33 & 6.75 & 5.97 & 2.72 & 2.89 & 1.06 \\
\hline MC_EH & 12.20 & 11.64 & 1.18 & 3.10 & 3.90 & 0.92 \\
\hline MC_AL & 11.54 & 11.41 & 1.49 & 3.30 & 3.48 & 0.50 \\
\hline
\end{tabular}

Table 6. Contact angle of samples

\begin{tabular}{ccccccc}
\hline \multirow{2}{*}{ Type } & \multicolumn{3}{c}{ Seokganju } & & & Seokrok \\
Samples & Original & Applied & Removed & Original & Applied & Removed \\
\hline SE_EH & 58.65 & 69.89 & 72.51 & 87.55 & 82.20 & 82.78 \\
\hline LP_AH & 65.51 & 69.52 & 85.86 & 83.27 & 82.35 & 97.40 \\
\hline LP_AL & 66.27 & 70.14 & 69.35 & 82.78 & 86.84 & 83.38 \\
\hline LP_ML & 19.70 & 69.72 & 101.6 & 45.76 & 89.96 & 111.82 \\
\hline MC_EH & 17.68 & 70.47 & 77.80 & 33.34 & 83.92 & 90.28 \\
\hline MC_AL & 23.54 & 75.12 & 78.62 & 51.66 & 89.70 & 91.51 \\
\hline
\end{tabular}


Table 7. Characteristics of experimental results

\begin{tabular}{|c|c|c|c|c|c|}
\hline \multirow[b]{2}{*}{ Samples } & \multirow{2}{*}{$\begin{array}{l}\text { Manufacture } \\
\text { available }\end{array}$} & \multirow[b]{2}{*}{ Workability } & \multicolumn{3}{|c|}{ Removed effect } \\
\hline & & & $\begin{array}{l}\text { Microscopic } \\
\text { observation }\end{array}$ & Color change & $\begin{array}{c}\text { Contact angle } \\
\text { change }\end{array}$ \\
\hline SE_EH & $\bigcirc$ & $\bigcirc \bigcirc$ & $\bigcirc$ & $\bigcirc$ & $\bigcirc$ \\
\hline LP_AH & $\triangle$ & $\bigcirc$ & $\bigcirc \bigcirc$ & $\bigcirc \bigcirc$ & $\triangle$ \\
\hline LP_AL & $\bigcirc$ & $\bigcirc$ & $\bigcirc$ & $\triangle$ & $\bigcirc$ \\
\hline LP_ML & $\bigcirc$ & $\bigcirc$ & $\bigcirc \bigcirc$ & $\bigcirc \bigcirc$ & $\triangle$ \\
\hline MC_EH & $\bigcirc \bigcirc$ & $\bigcirc \bigcirc$ & $\triangle$ & $\bigcirc$ & $\triangle$ \\
\hline $\mathrm{MC} \_\mathrm{AL}$ & $\bigcirc \bigcirc$ & $\bigcirc \bigcirc$ & $\triangle$ & $\bigcirc$ & $\triangle$ \\
\hline
\end{tabular}

treatment with the color difference down about 0.5 to 1 from the original sample. Seokrok, however, has shown a change of color with the average $\Delta \mathrm{E}$ of less than 1 after the application of the poulticing treatment with a color change similar to or even higher than that of the original sample. The MC sample, which have showed no significant change in color during the visual inspection have seen a wide range of change of color in Seokganju sample ranging from $\Delta \mathrm{E}$ 0.7 to 1.2 to $\Delta \mathrm{E} 1.5$ to 2.7 with no decrease in color difference compared with the original sample. The MC Seokrok sample have shown a low degree of color difference with the $\Delta \mathrm{E}$ of less than 1 with the color change similar to or higher than that of the original sample(Table 5).

\subsubsection{Results of contact angle measurements}

The average contact angles on the surface of Seokganju and Seokrok have been measured at 63.5 and 84.3, respectively. The contact angle of Seokganju following the application of Paraloid B72 has increased to 71.1 while that of Seokrok has remained similar at 83.8. The average contact angles of each of the sample for Seokganju and Seokrok with Paraloid B72 removed following the application of the poulticing treatment have shown to be 76.6 and 88.7, respectively, which are somewhat higher than the coating sample of Paraloid B72 in all samples(Table 6).

\section{DISCUSSION AND CONCLUSION}

Experiments were carried out to investigate the applicability of the poulticing with solvent to the removal of Paraloid
B72, which is a synthetic resin fixing agent used in Buddhist mural painting(Table 7).

Bentonite and sepiolite, which are clay types, are relatively easy to mix with solvent, it is good applicability for treatment of mural painting and has very low fluidity. However, at high MEK concentration, mixing was not good and viscosity was lower than other solvents. Gel type laponite, carbopol, and methyl cellulose were not gelated when mixed with high concentration of MEK. Particularly, the gel type poultices had a property of being mixed with a solvent after mixing with water.

As a result of checking the acidity characteristics of the poultices, most of the poultice materials were akaline and even after mixing with solvents were same. Carbopol was found to change acidity from neutral to acidic when mixed with high concentrations of acetone or ethanol. Therefore, it is deemed to be suitable to produce a low concentration of $25 \%$ or less when manufacturing a poultice pack with an organic solvent. The exact concentration of each poultice should be confirmed through preliminary experiments.

It should be considered that the drying of the poultices in the application of the poulticing. Experimental results show that clay type drying speed was faster than gel type when the same solvent was used. Therefore, clay type poultice is advantageous when applied for a relatively short time, and gel type poultice is suitable for a longer treatment. Generally, the poulticing is applied until the poultice is dried since the adsorption on the surface continues while the solvent is contained in the agent. However, the surface of the mural painting is soft compared with that of stone or ceramics, it is considered that the application of a short time 
wetting method is suitable for mural painting. In order to judge the proper applying time according to the removal object, the drying characteristics of the poultice and the solubility of the solvent should be confirmed in advance.

The solubility of the solvent was found to be effective even after mixing with the poultices, but the results were not uniform for each solvent and poultice. MEK showed the highest removal effect among the solvents, ethanol and acetone showed similar degree. The removal effect of ethanol was observed in both clay type and gel type, and acetone was removed only in gel form and clay type was ineffective in relative effect. It was considered that the effect of the gel type poultice was more effective than that of the clay type poultice which had relatively fast drying time because the time of application of organic solvent was longer. After applying, residuals were observed in carbopol sample, which was also confirmed by FT-IR analysis. It was concluded that carbopol were excluded from the coloring layer application test this time. However, if additional studies on the facing materials and methods are performed, it will be possible to find a way to complement for the residual of the poulticing.

Only the sample showing the characteristics suitable for mural painting were selected and applied to the colored layer test. As a result, LP_AH(50\%) and LP_ML(25\%) were found to have the highest removal efficiency. It was observed to absorb dissolved Paraloid B72. However, it is considered that remain were not absorbed into the poultice because a little of Paraloid B72 could not penetrate the rayon paper during the dissolving process. LP_AL and SE_EH showed that Paraloid B72 was solubilized by microscopic examination and measure of color change, but Paraloid B72 coating film still remained. MC_EH and MC_AL were also observed to dissolve the resin layer of Paraloid B72. However, when the results of the visual inspection and the chromaticity results are examined, it is considered that the laponite and sepiolite are more adsorbability. As a result of the contact angle measurement, the contact angle of the coated sample with the removed sample was measured at a similar or higher value. This is interpreted as showing that paraloid B72 penetrates into the colored layer and only the surface is removed when the poultice is applied. As we know from the results, it can be concluded that the physical damage of the colored layer would be very low if the selected organic solvent and pesticide application group are applied to the soil mural treatment.

It is believed that the above mentioned poultice conditions will be effective in removing the high concentrate Paraloid B72 was applied to earthen mural painting which the surface gloss is high.

In this study, it was confirmed that manufacturing of poultice are possible even solvent was mixed and the mixing ratio and drying characteristics were different depending on the type of poultice and solvent. Since the solubility varies depending on the concentration of the solvent and the characteristics of the poultice, it is necessary to select a suitable poultice and a solvent according to the state of the removal part. Finally, the application of the solvent directly to the colored layer may cause unpredictable damage such as coloring layer staining, discoloration, etc. Therefore, in order to apply this method, researches that apply Facing method under a variety of conditions should be performed more carefully.

\section{REFERENCES}

American Institute for Conservation of Art and Historic Works, 2018, Poulticing. http://www.conservation-wiki.com/wiki/ Poulticing (October 29, 2018)

Brajer, I., Shashoua, Y., Taube, M., Chelazzi, D., Baglioni, M. and Baglioni, P., 2014, The removal of aged acrylic coatings from wall paintings using micro emulsions. ICOM-CC 17th Triennial Conference, Melbourne, September 15-19.

Deurenberg, R.M.H., 2013, Examination and treatment of a set of Klismos chairs, Attr. To John and Hugh Finlay. American Institute of Conservation, 47(2), 97-117.

Garland, K.M, and Twilley, J., 2010, The scientific examination and re-treatment of an Egyptian limestone relief from the tomb of Ka-aper. Objects Specialty Group Postprints, 17, 113-132.

Han, K.S., 2002, The consolidation medium for the 
conservation of mud wall painting. Journal of Conservation Science, 11(1), 38-51. (in Korean with English abstract) Jin, B.H., Cho, J.Y., Park, J.Y., Han, S.H. and Kim, Y.S., 2017 , The study on removing paraloid B-72 from painting layer on mural of Mireukjeon hall at Geunsansa temple. MUNHWAJAE Korean Journal of Cultural Heritage Studies, 50(3), 104-125. (in Korean with English abstract)

Lee, H.S., Han, K.S. and Lee, S.J., 2013, A Study on painting layer fixative processing of mural paintings of buddhist temples in Korea. Journal of Conservation Science, 29(1), 81-92. (in Korean with English abstract)

Lee, J.W., Ham, C.H., Kim, S.D. and Lee, C.H., 2009, Removal methods of paint pollutants on the stone cultural heritage using poultices. Journal of Conservation Science, 25(4), 421-430. (in Korean with English abstract)

Lee, K.M., Han, K.S. and Lee, H.S., 2008, Assessing the effects of acrylic resin (Paraloid B-72) on buddhist mural-painting conservation -Focusing on outside mural paintings of Mireuk hall in Geumsan temple-. Conservation Studies, 29,
65-90. (in Korean with English abstract)

National Research Institute of Cultural Heritage, 2015, A study of damage diagnosis and fixative for mural paintings restoration techniques. National Research Institute of Cultural Heritage, Daejeon. (in Korean)

O'Hern, R. and Pearlstein, E., 2013, Label removal from deteriorated leather-bound books. Journal of the Institute of Conservation, 36(2), 109-124.

Park, D.W., Jang, S.Y., Nam, B.J., Ham, C.H. and Lim, S.T., 2011, Chemical cleaning of iron stains on ceramics. Journal of Conservation Science, 27(4), 345-356. (in Korean with English abstract)

Tong, D., 2013, The evaluation of modified laponite solvent-gel as a poultice in paper conservation. Art Conservation Program Queen's University, Kingston.

Warda, J., Brückle, I., Bezúr, A. and Kushel, D., 2007, Analysis of agarose, carbopol, and laponite gel poultices in paper conservation. Journal of the American Institute for Conservation, 46(3), 263-279. 